Objectives AXL is a receptor tyrosine kinase that is activated by GAS6. Overexpression of AXL is correlated with the glycolytic phenotype in metastatic lung cancer. Cancer cells preferentially convert glucose to lactate via glycolysis which promotes growth and survival. It is unknown whether inhibition of AXL can prevent glycolysis in endometrial cancer causing cell death. The aim of this study was to determine whether AVB-500 can increase sensitivity to paclitaxel through inhibition of glycolysis.

Methods Cell viability was performed with high-grade endometrial, chemo-resistant cell lines, ARK1 and PUC1. Cells were treated with paclitaxel (P) and with AVB-500+paclitaxel (AVB-500+P). Intraperitoneal ARK1 or PUC1 tumors were treated with vehicle, AVB-500, P, or AVB-500+P. Cell lysates were analyzed using the Jess system. A Seahorse Analyzer was used for glycolytic rate assays. Stable isotope tracing was used for in vivo metabolite abundance quantification.

Results We found that ARK1 and PUC1 cells had decreased viability when treated with $\mathrm{AVB}-500+\mathrm{P}$ than when treated with P alone. ARK1 and PUC1 in vivo IP models had significantly fewer tumors and decreased tumor weight when treated with AVB-500+P compared to P alone. Treatment with AVB$500+\mathrm{P}$ was found to decrease basal glycolysis in vitro through decreased AKT activation. Multiple glycolytic metabolites were decreased in the tumors of AVB-500 $+\mathrm{P}$ compared to treatment with $\mathrm{P}$ alone.

Conclusions We demonstrate that the addition AVB-500 to paclitaxel improves endometrial cancer chemo-sensitivity. We show that this therapeutic combination decreases basal glycolysis through reduced PI3K/AKT signaling. This provides a metabolic mechanism for increasing uterine cancer sensitivity to chemotherapy.

\section{EPV005/\#536 MOLECULAR ANALYSIS OF PRIMARY ENDOMETRIAL CANCER AND MATCHED LUNG METASTASES}

${ }^{1} S$ Gordhandas*, ${ }^{1} \mathrm{~A}$ Da Cruz Paula, ${ }^{2} \mathrm{E}$ Kertowidjojo, ${ }^{1} \mathrm{~K}$ Dessources, ${ }^{2} \mathrm{~A}$ Maroldi, ${ }^{2} \mathrm{E}$ Da Silva, ${ }^{2} \mathrm{~F}$ Derakhshan, ${ }^{1} \mathrm{~N}$ Abu-Rustum, ${ }^{1} \mathrm{~B}$ Weigelt. 'Memorial Sloan Kettering Cancer Center, Gynecology Service, Department of Surgery, New York, USA; ${ }^{2}$ Memorial Sloan Kettering Cancer Center, Pathology, New York, USA

\subsection{6/ijgc-2021-IGCS.72}

Objectives The molecular processes underpinning distant metastasis in endometrial cancer (EC) are not well understood. We sought to characterize the genomic alterations of primary ECs and matched lung metastases.

Methods Primary ECs, matched lung metastases, and normal tissue from two patients were subjected to whole-exome sequencing. Sequencing data were analyzed using validated bioinformatics tools.

Results In case 1 , sequencing analysis of the primary FIGO grade 2 EC and matched lung metastasis, which developed after 3 years, revealed 99 and 95 non-synonymous somatic mutations, respectively, of which 68 were shared. In addition to clonal shared PIK3R1 and PTEN mutations, we observed clonal shifts in the progression to lung metastatic disease, including a CTNNB1 G34V hotspot mutation, which was subclonal in the primary EC and became clonal in the metastasis. The primary dedifferentiated EC of case 2 was MSI-H due to MLH1 hypermethylation. Both the primary and synchronous metastatic lung tumor harbored a large number of somatic mutations (1217 and 1157, respectively), but only 227 were shared, including PIK3CA hotspot, PTEN frameshift, and MSH6 frameshift mutations. In case 2, clonal shifts were also observed in the progression from primary EC to lung metastasis, and multiple CTNNB1mutations were identified: a subclonal CTNNB1 G34R hotspot mutation in the primary EC, and a subclonal CTNNB1G33P hotspot and a clonal CTNNB1 G34E hotspot mutation limited to the metastasis.

Conclusions Clonal shifts, the accumulation of additional mutations and/or hotspot CTNNB1 mutations may play a role in the progression from primary EC to lung metastatic disease.

\section{EPV006/\#578 ROLE OF CHRONIC STRESS ON ANTI-TUMOR T- CELL RESPONSES IN OVARIAN CANCER}

${ }^{1} \mathrm{~A}$ Aquino-Acevedo*, ${ }^{2} \mathrm{H}$ Knochenhauer, ${ }^{1} \mathrm{M}$ Ortiz-León, ${ }^{1} \mathrm{Y}$ Rivera-López, ${ }^{1} \mathrm{M}$ BonillaClaudio, ${ }^{2} \mathrm{R}$ Previs, ${ }^{1} \mathrm{G}$ Armaiz-Pena. ${ }^{1}$ Ponce Health Sciences University, Department of Basic Sciences (pharmacology), Ponce, Puerto Rico; ${ }^{2}$ Duke University School of Medicine, Obstetrics and Gynecology, Durham, USA

\subsection{6/ijgc-2021-IGCS.73}

Objectives A cancer diagnosis increases stress hormones and leads to altered psychological states. Work from our team suggests that chronic stress promotes an increased inflammatory response. Preliminary data show an altered $\mathrm{CD} 4+/ \mathrm{CD} 8+\mathrm{T}-$ cell ratio and a heterogeneous expression of exhaustion markers in patients with high-grade serous ovarian cancer (HGSOC). Therefore, we hypothesized that chronic stress results in loss of effector T-cell response and increased exhaustion.

Methods We obtained ascites samples from 66 patients with HGSOC and measured cytokine levels using a comprehensive cytokine/chemokine magnetic bead panel. Metanephrine (an epinephrine metabolite) levels from ascites were measured by ELISA. CD8 + T-cells isolated from OC patient ascites were stimulated with epinephrine and flow cytometry was used to measure co-expression of CD38 activation marker and Granzyme B, an essential mediator of CD8 + T-cell killing capacity. Results Showed a significant increase in inflammatory cytokines in chemo-resistant and recurrent tumors: Eotaxin $(p \leq 0.002)$, IL-6 ( $\mathrm{p} \leq 0.003)$, and IL-7 ( $\mathrm{p} \leq 0.009)$. Metanephrine, was positively correlated with pro-tumoral and inflammatory cytokines: SCD40L $(p=0.032)$, FGF-2 $(p=0.033)$ and MIP1a $(\mathrm{p}=0.03)$. Ascites-derived CD8 + T-cells treated with epinephrine, showed a decreased co-expression CD38 and Granzyme B $(p=0.004)$. These results suggest a role for stress hormones in T-cell activity suppression.

Conclusions Chemo-resistant and recurrent tumors were associated with increased pro-inflammatory cytokines. Similarly, high metanephrine levels correlated with higher pro-tumoral cytokines. Epinephrine stimulation decreased CD8 + T-cell function in ascites of HGSOC patients. These data suggest a role for stress in immunosuppression and may impact efficacy of therapies that aim to restore T-cell function. 